\title{
Migration of gastric cancer is suppressed by recombinant Newcastle disease virus (rL-RVG) via regulating a7-nicotinic acetylcholine receptors/ERK- EMT
}

Xuefeng $\mathrm{Bu}^{1 \dagger}$, Anwei Zhang ${ }^{2,3 \dagger}$, Zhengwei Chen ${ }^{3}$, Xuanfeng Zhang ${ }^{3}$, Riting Zhang ${ }^{3}$, Chaoyun Yin ${ }^{4}$, Jie Zhang ${ }^{1}$, Yao Zhang ${ }^{3}$ and Yulan Yan $^{5^{*}}$ (D)

\begin{abstract}
Background: Nicotinic acetylcholine receptors (nAChRs) have been reported to be overexpressed in malignancies in humans and is associated with tumorigenesis and cell migration. In previous studies of gastric cancer, alpha7 nicotinic acetylcholine receptor (a7-nAChR) overexpression leads to epithelial-mesenchymal transition (EMT) and promotes the migration of gastric cancer cells. Recombinant avirulent LaSota strain of Newcastle disease virus (NDV) expressing the rabies virus glycoprotein (rL-RVG) may promote apoptosis of gastric cancer cells and reduces the migration of lung cancer metastasis. However, whether rL-RVG inhibits migration of gastric cancer cells and what the underlying functional mechanism is remains unknown.

Methods: The gastric cancer cell lines BGC and SGC were randomly divided into 3 groups: rL-RVG, NDV and Phosphate Buffered Solution (PBS) control groups. Furthermore,we adopted ACB and MLA,a7nAChR-siRNA for the overexpression and silencing of a7-nAChR.Corynoxenine was used for inhibiting the MEK-ERK pathway. Western blot, Immunofluoresce,cell proliferation assays,cell migration analyses through wound-healing assays and Transwell assays were used to explore the underlying mechanisms. A mouse xenograft model was used to investigate the effects of rL-RVG,NDV on tumor growth.

Results: In this study, our findings demonstrate that rL-RVG suppressed the migration of gastric cancer cells and reduced EMT via a7-nAChR in vitro. Furthermore rL-RVG decreased the phosphorylation levels of the MEK/ERK signaling pathway such as down-regulating the expression of P-MEK and P-ERK. Additionally, rL-RVG also reduced the expression level of mesenchymal markers $\mathrm{N}$-cadherin and Vimentin and enhanced the expression of the epithelial marker E-cadherin. Lastly, rL-RVG inhibited nicotinic acetylcholine receptors (nAChRs) to suppress cell migration and epithelial to mesenchymal transition (EMT) in gastric cell. We also found that rL-RVG suppresses the growth of gastric cancer subcutaneous tumor cells in vivo.
\end{abstract}

Conclusion: rL-RVG inhibits a7-nAChR-MEK/ERK-EMT to suppress migration of gastric cancer cells.

Keywords: Recombinant Newcastle disease virus, Rabies virus glycoprotein, a7-nAChR, Epithelial-mesenchymal transition, Migration, Gastric cancer, ERK

\footnotetext{
* Correspondence: ylyan2005@163.com

† Xuefeng Bu and Anwei Zhang contributed equally to this work.

${ }^{5}$ Department of Respiratory Medicine, Affiliated People's Hospital of Jiangsu

University, DianLi Road No.8, Zhenjiang 212002, JiangSu, China

Full list of author information is available at the end of the article
}

(c) The Author(s). 2019 Open Access This article is distributed under the terms of the Creative Commons Attribution 4.0 International License (http://creativecommons.org/licenses/by/4.0/), which permits unrestricted use, distribution, and reproduction in any medium, provided you give appropriate credit to the original author(s) and the source, provide a link to the Creative Commons license, and indicate if changes were made. The Creative Commons Public Domain Dedication waiver (http://creativecommons.org/publicdomain/zero/1.0/) applies to the data made available in this article, unless otherwise stated. 


\section{Background}

Stomach cancer is the fifth most common cancer and the third major cause in cancer-related deaths in the word [1]. Moreover, the mortality of stomach cancer is the second highest among all cancer related deaths in China according to the statistics of 2016 [2]. It is difficult to detect the early stages of gastric cancer and there are an overwhelming number of patients who are diagnosed late [3]. Therefore, the study of the mechanisms of cancer cell migration mechanisms during stomach cancer is of great significance for new drug discoveries and for the development of effective treatments for gastric cancer. Currently, the main treatment of gastric cancer consists of surgical treatment, targeted therapy, immunotherapy, radiotherapy and chemotherapy. However, these methods are accompanied by poor patient prognosis with advanced gastric cancer and therefore a novel treatment is urgently needed for people affected by this devastating disease.

Nicotinic acetylcholine receptors (nAChRs) are a type of ligand-gated ion channel proteins, and their expression is not only found on neuronal cells but also in non-neuronal cells including gastric cancer cells $[4,5]$. Alpha7 nicotinic acetylcholine receptor $(\alpha 7-n A C h R)$ is a member of the family of nAChRs and is widely expressed on stomach epithelial cells $[6,7]$. It has been reported that the activation of $\alpha 7-n A C h R$ plays an important role in the proliferation and migration of cancer cells. The extracellular signalregulated kinase (ERK) signaling pathway is involved in a variety of diseases and activated by several stimuli including $\alpha 7-n A C h R[8]$.

Epithelial to mesenchymal transition (EMT) is the original biological step required for the invasion of cancer cells and metastasis into tissues. A hallmark of EMT includes the presence of mesenchymal markers such as Ncadherin, Vimentin and the epithelial marker E-cadherin $[9,10]$. EMT is of great significance for tumor migration [11], and a recent study also suggested that alpha 7nAChR overexpression could enhance EMT to promote proliferation and migration of gastric cancer cells [11]. Moreover, it was reported that nicotine could promote EMT to induce migration of cancer cells via regulating of the alpha 7-nAChR/MEK/ERK pathway [12].

Alpha 7-nAChR may be a potential therapeutic key point for the treatment of stomach cancer. Previous studies have also demonstrated that the recombinant avirulent NDV LaSota strain, expressing the rabies virus glycoprotein (rL-RVG), could induce the apoptosis of gastric cancer cells as well as suppress the migration of lung cancer cells by regulating $\alpha 7-$ nAChR [13, 14]. However, it still remains unclear whether rL-RVG could also suppress the migration of gastric cancer and its underlying mechanisms. In this study, we explored if rL-RVG could suppress the migration of gastric cancer cells via regulating $\alpha 7$ nAChR/ERK signaling and EMT.

\section{Methods \\ Materials}

The rL-RVG, NDV, anti-RVG antibody and anti-NDV antibody were stored at $-80^{\circ} \mathrm{C}$ supplied by the Harbin Veterinary Research Institute (Harbin, China). The human gastric cancer cell lines SGC7901 and BGC were purchased from the Cancer Cell Repository (Shanghai Cell Bank,201611-20) and the American Type Culture Collection (Manassas,VA,USA). The cell line has been authenticated by STR (short tandem repeats, STR) and has been tested for mycoplasma contamination (Additional file 1). Nude mice were purchased from the. Animal Experiment Center of Yangzhou University (Yangzhou, China). Methyllcaconitine citrate hydrate (MLA) $(5 \mathrm{mg} / \mathrm{mL})$ which worked as a specific competitive $\alpha 7-n A C h R$ antagonist was purchased from Santa Cruz (California, USA). Acetylcholine bromide (ACB) $(5 \mathrm{mg} / \mathrm{mL})$,an acetylcholine agonist, was obtained from Sigma-Aldrich (St. Louis, MO, USA). In addition, small interfering RNA (si-RNA) for $\alpha 7-n A C h R$ was purchased from RiboBio (GuangZhou, China). Corynoxenine (10 mM $1 \mathrm{~mL}$ in DMSO),the inhibitor of the MEK-ERK pathway, was purchased from MedChemExpress (MCE,USA). Rabbit polyclonal anti- $\alpha 7 \mathrm{nAChR}$ was purchased from Abcam (London, UK); rabbit polyclonal anti-MEK1/2, anti-PMEK1/2, anti-ERK1/2, anti-P-ERK1/2 and anti-snail were purchased from Cell Signaling Technology (CST, USA); Mouse monoclonal anti-N-Cadherin, E-Cadherin and Vementin were purchased from Boster (WuHan,China).

\section{Cell culture reagents, viruses and treatment}

The human cell lines BGC and SGC were cultured in RPMI 1640 supplemented with 10\% fetal bovine serum, penicillin $(100 \mathrm{U} / \mathrm{mL})$, and streptomycin $(100 \mu \mathrm{g} / \mathrm{mL})$ in a humidified incubator $\left(37^{\circ} \mathrm{C}, 5 \% \mathrm{CO}_{2}\right)$. In addition, when BGC and SGC cell lines reached their logarithmic proliferation phase of up to $80 \%$ confluency, they were then sub cultured or used for experiments. The cultured cell lines were randomly divided into rL-RVG, NDV and PBS groups, along with the MLA, ACB, si-RNA of $\alpha 7-$ $\mathrm{nAChR}$ and corynoxenine pretreatment groups.

\section{CCK-8 assay}

The viability of infected BGC and SGC were monitored by performing a CCK-8 assay. BGC and SGC were harvested, pelleted by centrifugation and counted using a blood counting chamber. Cells $\left(6 \times 10^{3}\right)$ were then seeded into 96-well plates and grown in media containing varying dilution titers of rL-RVG or NDV $24 \mathrm{~h}$. The CCK-8 reagent was added into each well incubated for another $4 \mathrm{~h}$. Lastly, the color of the media changed in each well and was measured at $450 \mathrm{~nm}$ using a spectrophotometer. 


\section{Clonogenic survival assay}

BGC and SGC were seeded into 6-well plates (1000 cells per well) and then infected with rL-RVG, NDV at a multiplicity of infection of 10 or incubated with PBS for $24 \mathrm{~h}$. After incubation for 10 days, the cells were fixed with absolute ethyl alcohol for $30 \mathrm{~min}$ and stained for 1 $\mathrm{h}$ with crystal violet $(0.2 \%)$ to visualize cell colonies. Each individual experiment was repeated three times.

\section{Wound-healing assay}

BGC and SGC were added into 6-well plates and randomly divided into rL-RVG, NDV and PBS treated groups in which cell were infected by rL-RVG,NDV or treated with PBS for $24 \mathrm{~h}$. Groups pretreated with ACB, MLA, siRNA or corynoxeine for $24 \mathrm{~h}$ were also set up. When cells reached $80 \%$ confluency, cell monolayers were disrupted with a $10 \mu \mathrm{L}$ pipette tip after $24 \mathrm{~h}$ and their wound healing ability was determined by microscopy.

\section{Migration assay}

To carry out migration assays in 24-well transwell plates with polycarbonate membranes $(8.0 \mu \mathrm{m}$ pore size; Costar, MA, USA) were used for in-vitro experiments according to the manufacturer's protocol. In short, the upper chamber of the filter inserts contained serum free medium with $1 \times 10^{5}$ cells belonging to one of the treatment groups: $\mathrm{rL}$ RVG, NDV, PBS, MLA, ACB, si-RNA of $\alpha 7-n A C h R$ or corynoxenine. Meanwhile, the lower chamber was filled with $600 \mu \mathrm{L} 10 \%$ serum media. After incubation at $37^{\circ} \mathrm{C}$ in $5 \% \mathrm{CO}_{2}$ for $24 \mathrm{~h}$, the media in the lower chamber were collected and then non migrating cells attached to the membrane of the upper chamber surface were scrubbed. Finally, cells on the bottom wells were stained with $0.1 \%$ crystal violet for $20 \mathrm{~min}$,and then washed with PBS for 3 times. Lastly, the migrating cells were counted under the microscope and analyzed by using Image-J software. (National Institutes of Health,USA).

\section{Western blot analysis}

After pre-treatment with MLA or ACB or si-RNA of $\alpha 7-$ $\mathrm{nAChR}$ or corynoxenine for $12 \mathrm{~h}$, cells were infected for $24 \mathrm{~h}$ with either rL-RVG,NDV or PBS and then washed with ice-cold PBS for 3 times and lysed by using the lysis buffer RIPA containing $1 \mathrm{mM}$ PMSF for $30 \mathrm{~min}$ on ice. Next the lysates were collected and the protein concentrations were quantified using a BCA kit (Thermo Fisher Scientific, USA). Equal quantities of protein were separated by using a $10 \%$ SDS-PAGE and the proteins were then transferred to polyvinylidinedifluoride (PVDF) membranes (Bio-Rad Laboratories). The membranes were then blocked with 5\% BSA in Tris-buffered saline containing $0.1 \%$ Tween 20 (TBST, at $\mathrm{pH} 7.5$ ) for about $2 \mathrm{~h}$ at room temperature before washing them with TBST for 15 min for 3 times. Next the membranes were incubated with antibodies at $4{ }^{\circ} \mathrm{C}$ overnight with the following antibodies: anti- $\alpha 7 \mathrm{nAChR}$, anti-P-MEK, antiMEK, anti-P-ERK, anti-ERK, anti-E-cadherin, anti-Ncadherin and anti-Vementin. Proteins were detected with HRP-conjugated secondary antibodies for $1 \mathrm{~h}$ at room temperature. The protein bands were visualized with a Typhoon 9400 variable mode imager (Amersham Biosciences, UK) using chemiluminescence (ECL Plus Substrate, Thermo Fisher Scientific, USA).

\section{Immunofluorescent assay}

BGC and SGC cells were added into 24-well plates and fixed with $4 \%$ paraformaldehyde for $2 \mathrm{~h}$ at room temperature. Cells were then permeabilized with $0.5 \%$ TritonX-100 in PBS for $10 \mathrm{~min}$ and blocked with 5\% BSA for $1 \mathrm{~h}$. Next the cells were washed 3 times with PBS for $5 \mathrm{~min}$ before incubating them with anti-P-ERK at $4{ }^{\circ} \mathrm{C}$ overnight. On the next day, the secondary antibody was used to stain cells in order to be detected for immunofluorescence microscopy.

\section{Xenografts}

After infection of BGC, SGC cells with rL-RVG, NDV and incubation with PBS for $24 \mathrm{~h}$, the cells were subcutaneously injected into axillary subcutaneous tissues of adult female athymic-nude mice which were randomly selected for the treatment with either rL-RVG,NDV or PBS and housed under specific pathogen free conditions. The size of subcutaneous tumors that developed were measured 2 weeks post treatment using the following calculation: $\mathrm{V}=\mathrm{W}^{2} \mathrm{~L} 0.5$ ( $\mathrm{V}$ is volume, $\mathrm{W}$ is width and $\mathrm{L}$ is length). In this experiment,we used $2.5 \%$ pentobarbital sodium $(50 \mathrm{mg} / \mathrm{kg})$ to anaesthetize the athymic-nude mice by intraperitoneal injection. At last,we used 2.5\% pentobarbital sodium $(200 \mathrm{mg} / \mathrm{kg})$ to overdose the rats with anesthetic to euthanize them.

\section{Statistical analysis}

All collected experimental data is presented as mean \pm SD. A Student's t-test or one -way ANOVA with Bonferroni post-test was used to calculate statistical significance using the GraphPad Prism7.0 software (La Jolla, CA, USA). A $p$-value of $P<0.05$ or $P<0.01$ was considered statistically significant. Each experiment was conducted independently and repeated at least 3 times.

\section{Results}

RVG and NDV protein expression in gastric cancer cells

To investigate the mechanism of rL-RVG suppressing the migration of gastric cancer cells, we first analyzed the expression of rL-RVG and NDV proteins in gastric cancer. Previous studies show that lung cancer cell exhibit a stable expression of RVG and NDV proteins by PCR, Western blot and immunofluorescence microscopy 

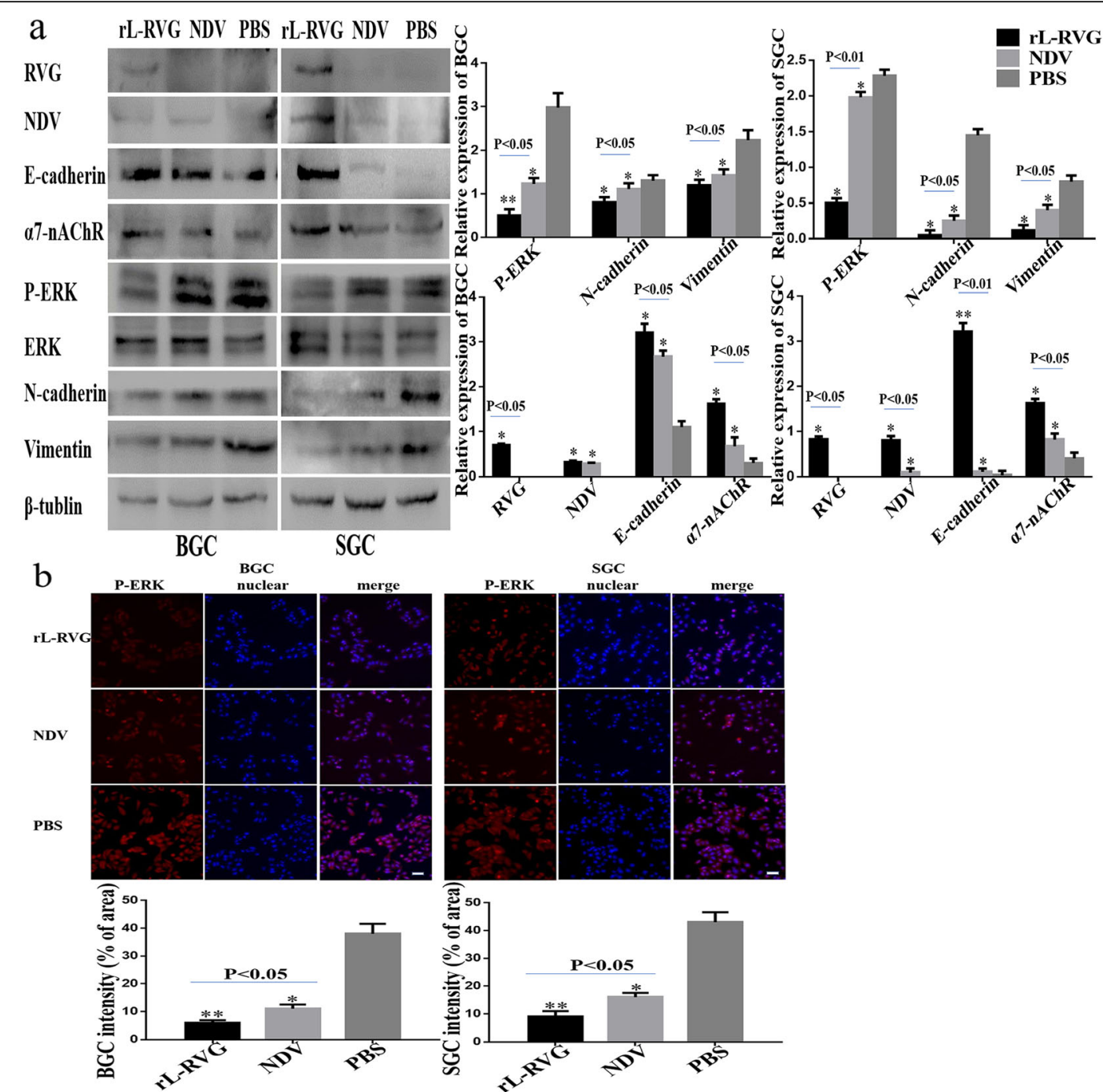

C
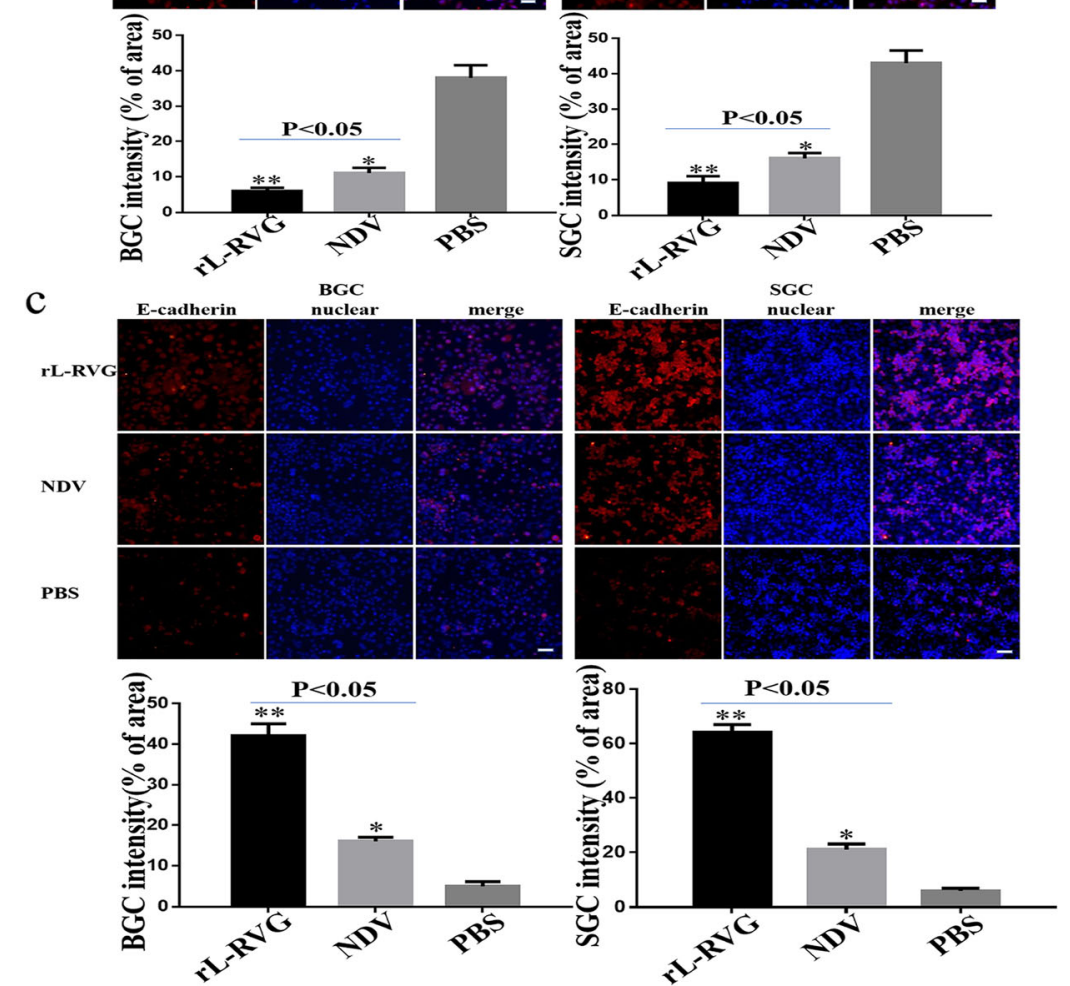

Fig. 1 (See legend on next page.) 
(See figure on previous page.)

Fig. 1 Expression of RVG, NDV, a7-nAChR, MEKJERK signaling pathway and epithelial/mesenchymal markers proteins in infected BGC and SGC cells. a Western blot analysis of RVG, NDV, a7-nAChR, MEK/ERK signaling pathway and epithelial/mesenchymal proteins. b Immunofluorescence analysis of P-ERK. c Immunofluorescence analysis of EMT protein markers E-cadherin. BGC and SGC cells were infected with either rL-RVG, NDV and PBS for $24 \mathrm{~h} .{ }^{*} P<0.5,{ }^{*} P<0.01$. (rL-RVG Vs NDV,rL-RVG vs NDV and PBS groups, respectively, Bar $=25 \mu \mathrm{m}$ )

[15]. In our study, we used Western blot to analyze both RVG and NDV protein expression in virally infected gastric cancer cells and found that RVG proteins were only expressed in the rL-RVG group while the expression of NDV proteins was expressed in both the rL-RVG and NDV group (Fig. 1a).

\section{rL-RVG suppressed the proliferation and migration of gastric cancer cells}

The formation of metastasis are a big challenge for the treatment of cancer. To study metastasis migration we used a transwell-based wound healing assay to monitor the influence of viruses on gastric cancer cells migration. After infecting cells with rL-RVG or NDV for $24 \mathrm{~h}$, we observed that rL-RVG and NDV both reduced the migration of gastric cancer cells compared to the PBS treated control group. Of note is that the inhibitory migration was stronger in rL-RVG treated cells compared to the NDV group (Fig. 2a-b). Moreover, we found that rL-RVG inhibited the migration of both SGC and BGC cells and we selected SGC cells for further analysis in subsequent experiments.

To determine the viability of gastric cancer cells, SGC and BGC cells were infected with rL-RVG or NDV for 24 $\mathrm{h}$ and analysed by using a CCK8 assay. rL-RVG and NDV both suppressed cell proliferation in a concentrationdependent manner but overall rL-RVG had a stronger inhibitory effect on proliferation compared to NDV and the PBS control group. rL-RVG and NDV were diluted to $10^{3}$ and $10^{2}$ respectively, the viability of SGC and BGC cells was higher than $80 \%$, and the viral titer was approximately $10^{9.8}$ EID $_{50} / \mathrm{mL}$ (Fig. 2c-d).

\section{The role of a7-nAChR in the process of rL-RVG regulated migratory abilities of gastric cancer cells}

The expression of $\alpha 7-n A C h R$ in the rL-RVG and NDV groups was higher compared with the PBS group using Western Blot analysis (Fig. 1a). However the cell migratory ability in both the rL-RVG and NDV group was suppressed compared with the PBS control group (Fig. 1a). rL-RVG suppressed the migration more potently compared with the NDV and PBS groups (Fig. 1a, Fig. $2 \mathrm{a}-\mathrm{b})$. Further exploration regarding the role of $\alpha 7$ $n A C h R$ on rL-RVG on the suppression of the migration of gastric cancer cells is necessary and the following treatments were performed.

MLA, an antagonist of $\alpha 7-n A C h R$ was used to pre-treat SGC cells for $24 \mathrm{~h}$ before infecting them with virus. Our result show that $\alpha 7-\mathrm{nAChR}$ expression was inhibited in a competitive manner and moreover, the migration of SGC cells was more suppressed in the MLA pre-treatment groups compared to the not pre-treated groups as shown in our migration and wound healing assays. This result suggests that rL-RVG may suppress cell migration through the $\alpha 7-n A C h R$ pathway (Fig. 4a-b).

To further verify the role of $\alpha 7-n A C h R$ in rL-RVGinduced cell migration, we used small interfering RNA methods to knock down the expression of $\alpha 7-n A C h R$. We found that our results were consistent with the groups pre-treated with MLA. Therefore, rL-RVG may play a role as competitive antagonist of $\alpha 7-n A C h R$ to inhibit the migration of SGC cells (Fig. 5a-b).

In support of these results we found that $\mathrm{ACB}$, an agonist of $\alpha 7-n A C h R$ stimulates the expression of $\alpha 7-n A C h R$ (Fig. 3a-b). In contrast to this, we got the opposite result when comparing our result with MLA or si-RNA pretreated cells. Thus, rL-RVG suppresses the migration of SGC cells by inhibiting $\alpha 7-n A C h R$ competitively.

\section{MEK/ERK signaling pathway was involved in rL-RVG- lowing migration of gastric cancer cell}

The MEK/ERK pathway is downstream of the signaling pathway of $\alpha 7-n A C h R$ and is involved in tumorigenesis [8]. The role of the extracellular signal-regulated kinase (ERK) signaling pathway in the rL-RVG-induced suppression of cell migration during gastric cancer remains unclear. Western blot analysis showed that rL-RVG induces the down-regulation of phosphorylation levels of ERK1/2 when $\alpha 7-n A C h R$ expression was blocked by rL-RVG (Fig. 1a). Furthermore the migratory ability of gastric cancer cells was also decreased. Consistent with these results we showed that the expression level of P-ERK was downregulated after being infected with either rL-RVG or NDV but was lower in the rL-RVG group compared with the NDV and PBS blank control group using immunofluorescence microscopy (Fig. 1a,c and Fig. 2a-b).

To further support our results we showed that the levels of ERK1/2 phosphorylation was lower compared with the non-pretreated groups after pretreatment with MLA or siRNA of $\alpha 7-n A C h R$ by Western blot and lmmunofluorescence (Fig. 4a,c and Fig. 5 a,c). Our migration and wound healing assay revealed that the migratory ability of SGC cells in the pretreated group decreased more compared to non pretreated cells (Fig. $4 \mathrm{~b}$ and Fig. 5b). To clarify whether rLRVG modulates the MEK/ERK signaling pathway to suppress cell migration, corynoxenine, an inhibitor of the MEK/ 

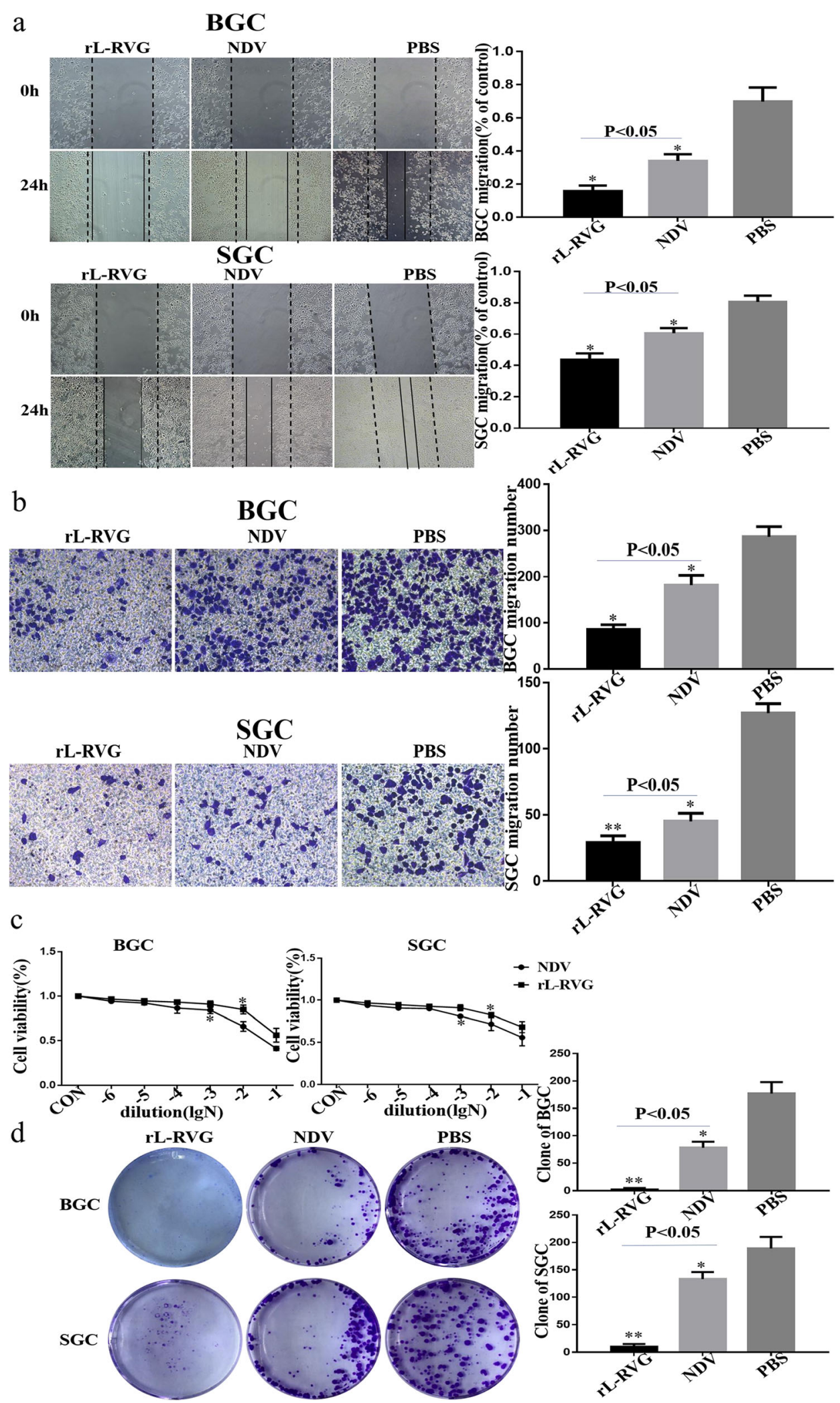

Fig. 2 (See legend on next page.) 
(See figure on previous page.)

Fig. 2 rL-RVG suppresses the proliferation and migration of BGC and SGC cells. a Healing and $\mathbf{b}$ Transwell assays were used to monitore the migration of BGC and SGC cells infected with rL-RVG, NDV and PBS, respectively. $\mathbf{c}$ Influence of different rL-RVG, NDV dilution titers on the viability of BGC and SGC cells. $\mathbf{d}$ The clonogenic activity of BGC and SGC cells after infection with rLRVG and NDV at a multiplicity of infection of ten. Colony formation was attenuated in the rL-RVG group. ${ }^{*} P<0.5,{ }^{* *} P<0.01$. (rL-RVG VS NDV and PBS groups, respectively)

ERK pathway, was used to pre-treat gastric cancer cells (Fig. 6a-d). We obtained comparable results in the groups treated with MLA and si-RNA of $\alpha 7-n A C h R$ as described above. However, opposite results were obtained when cells were pre-treated with ACB (Fig. 3a-d). These results indicate that rL-RVG attenuates the activation levels of MEK/ERK pathway via blocking $\alpha 7-n A C h R$ by competition.

\section{rL-RVG reduced EMT by regulating a7-nAChR}

Our study found that the protein expression level of Ecadherin was increased while $\mathrm{N}$-cadherin, and Vimentin were decreased in the rL-RVG or NDV groups compared with the PBS treated control group (Fig. 1a and c). Furthermore, the groups pre-treated with MLA showed that the expression of E-cadherin was lower and the expression of N-cadherin and Vimentin was higher compared with the non-pretreated groups using western blot analysis (Fig. 4a, d). We found similar results in the $\alpha 7-$ nAChR si-RNA pretreated group (Fig. 5a and d) and in the corynoxenine treated group (Fig. $6 \mathrm{a}$ and $\mathrm{d}$ ). In addition,the opposite result was obtained in the ACB pretreated groups when compared with the MLA or siRNA pretreated groups.

\section{rL-RVG inhibits subcutaneous growth of gastric cancer cells}

A tumor-bearing mouse model was established to confirm the antitumor effect of rL-RVG in vivo. Our results show that the tumor size of nude mice infected with rLRVG and NDV was smaller than in the PBS group, and within the treated groups the tumor size in the rL-RVG group was smaller than in the NDV group (Fig. 7).

\section{Discussion}

With the development of new drug treatments, the incidence of gastric cancer has declined rapidly over the recent years [16] but still results in over 1,000,000 new cases and an estimated 783,000 deaths in 2008 (equating to 1 in every 12 deaths globally) [1]. Early diagnosis and early treatment of gastric cancer is still a major challenge to reduce the mortality of gastric cancer [18]. Tumor metastasis is the main cause of death in patients with gastric cancer. Therefore it is important to understand how to reduce metastasis formation and to find treatment options for curing advanced-stage gastric cancer. In our study, recombinant avirulent NDV LaSota strain expressing the rabies virus glycoprotein rL-RVG did not only suppress migration of gastric cancer cells such as
BGC and SGC cells in vitro, but also inhibited growth of subcutaneous tumor in nude mice in vivo.

NDV replicates selectively and destroys tumor cells without damaging healthy cells $[17,18]$. Thus, selective killing of cancer cells is a highly beneficial while side effects are kept to a minimum. The 198-214 amino acid sequence of Rabies virus glycoprotein (RVG) is highly homologous with the 30-56 amino acid sequence in $\lambda$ bungarotoxin, which binds nAChRs. Therefore rL-RVG and $\lambda$-bungarotoxin have similar inhibitory effects on nAChRs.rL-RVG did not have oncolytic effects but inhibited nAChRs. Propranolol is a nonselective, competitive antagonist of beta adrenergic receptors. In this study,rL-RVG exhibited a similar mechanism of function like to Propranolol and played a role as competitive antagonist of $\alpha 7-n A C h R$ after infecting gastric cancer cell lines such as BGC and SGC.

nAChR consists of five subunits and assembles into heteromeric or homomeric pentamers. $\alpha 7-n A C h R$,another type of $\mathrm{nAChR}$, had a positive effect on cancer cell migration [19]. Nicotine and NNK act as agonist of $\alpha 7$ $\mathrm{nAChR}$ to facilitate the migration of gastric cancer cells $[11,20]$ and indirectly activate ERK signaling through promoting the release of epidermal growth factor (EGF) and trans-activation of EGF receptors [21]. Moreover, the ERK signaling pathway also has influence on EMT that may regulate expression of mesenchymal and epithelial repressor genes [22]. In our study, we suggest that rL-RVG lowers the phosphorylation levels of ERK signaling and decrease EMT in SGC and BGC cells, indicating that gastric cancer cell migration is associated with the MEK-ERK-EMT signaling pathway.

It is of great significance to activate EMT for invasion and metastasis of gastric cancer cells [23]. An aberrant EMT activation typically results in the transformation of epithelial cells into mesenchymal cells and leads to phenotypic changes such as the loss of cell-cell adhesion, cell polarity and acquisition of migratory and invasive properties of cells. Cadherin is a significant component of adherent cell junctions. On one hand, aberrant activation of EMT transformed E-cadherin to N-cadherin, which is typically found in mesenchymal cells and promotes the formation of adhesions between cells and the stroma [24]. On the other hand, Vimentin is a widespread mesenchymal intermediate filament which results in adhesion and migration of activated cells [25] and is key for the aberrant EMT activation in gastric cancer [26]. Our previous studies suggest that rL-RVG promotes apoptosis of gastric cancer cells [13, 27] but the 


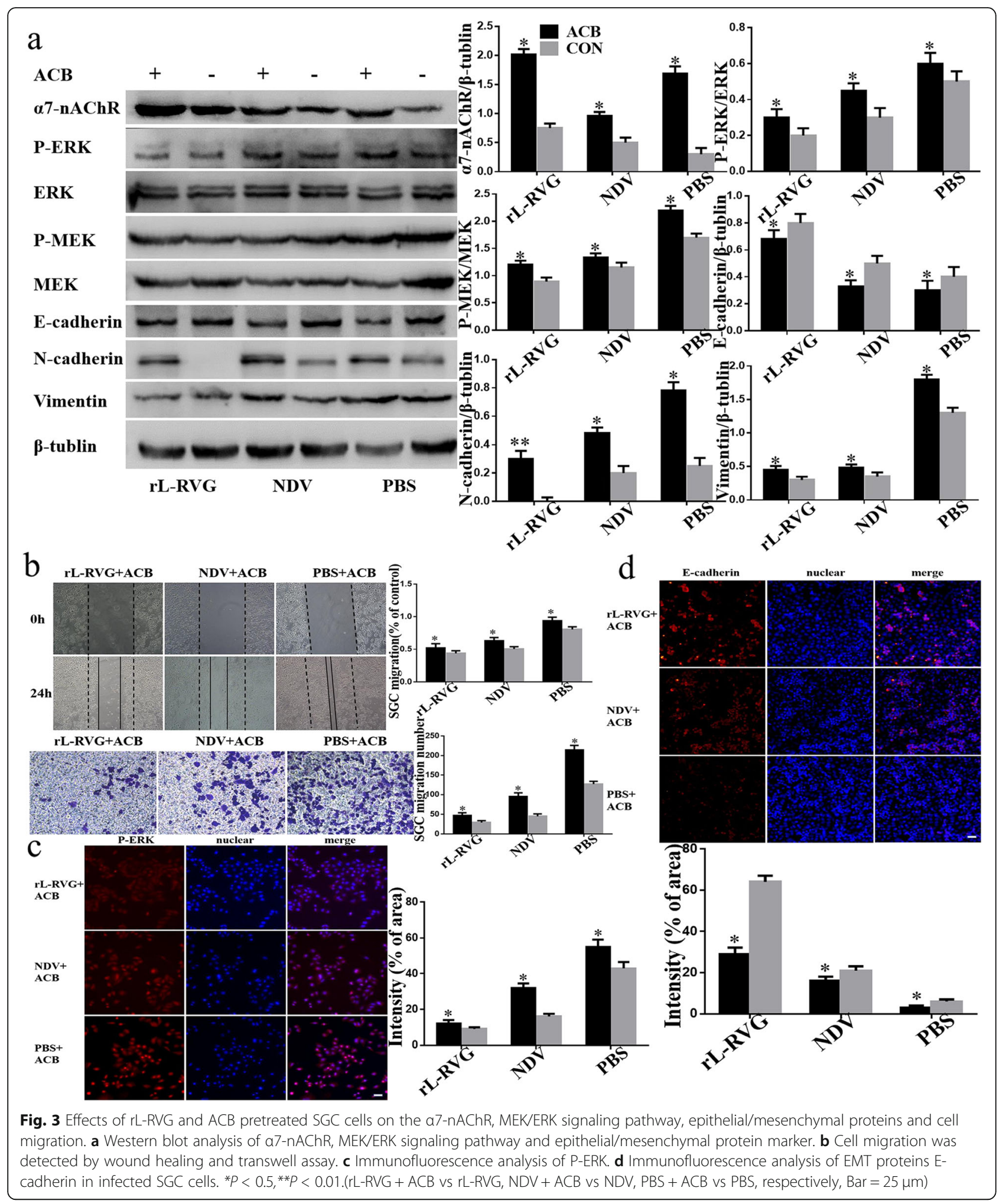

effect of rL-RVG on gastric cancer migration and its underlying mechanism remain unknown. In this study, our results show for the first time that gastric cancer cell migration is suppressed after infection of gastric cancer cells with rL-RVG through competitive inhibition of $\alpha 7-n A C h R$. This resulted in the decreased expression of mesenchymal markers including $\mathrm{N}$ cadherin and vimentin and increased levels of $\mathrm{E}$ - 


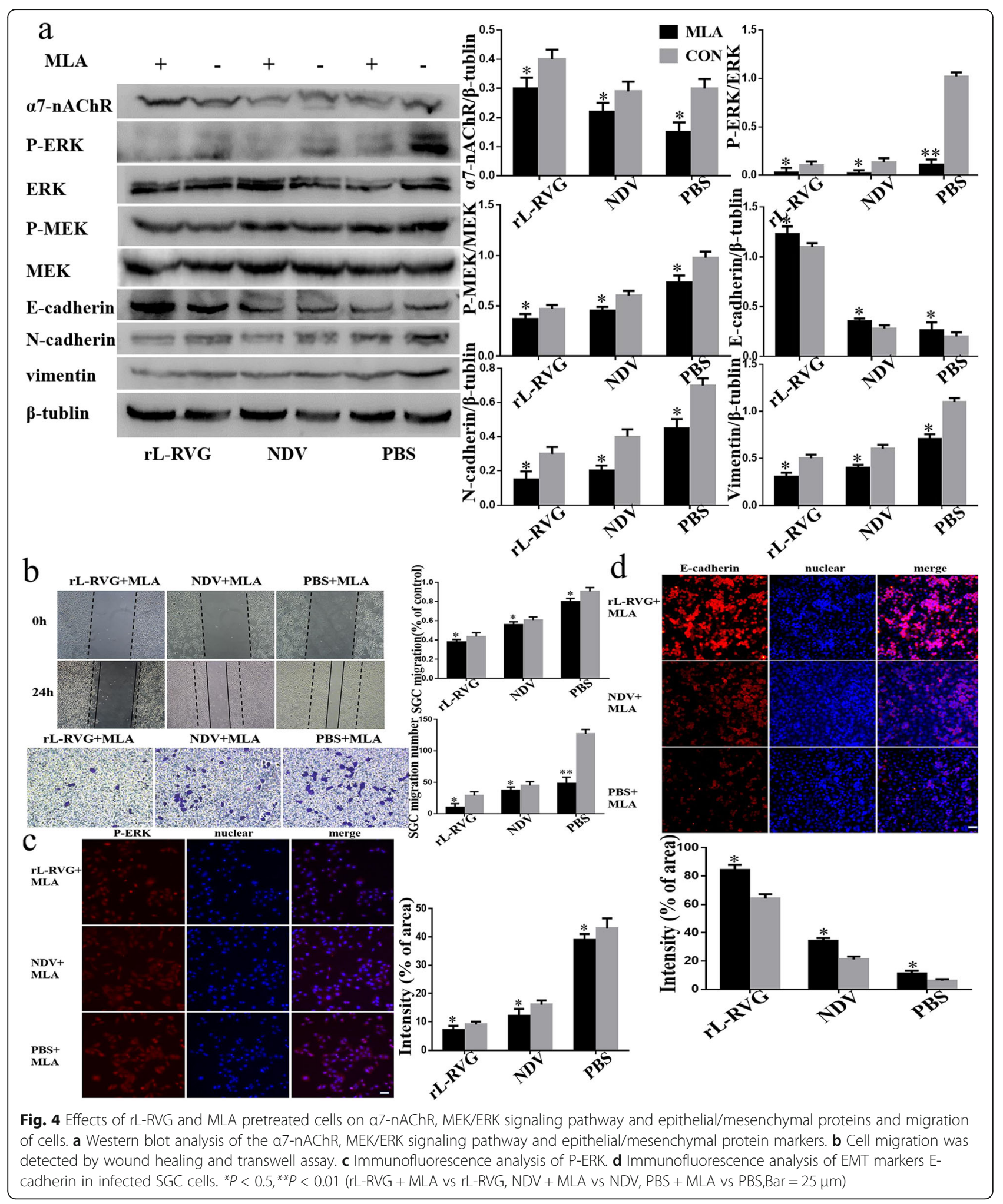

cadherin. Nicotine promotes the migration of gastric cancer cells via the $\alpha 7-n A C h R$ pathway [11]. Thus, we hypothesized that rL-RVG might suppress the migration of cancer cells via $\alpha 7-n A C h R$ by using
ACB, MLA and si-RNA of $\alpha 7-n A C h R$ to pre-treat SGC cells.

Previous studies suggest that the ERK signaling pathway plays an important role in the proliferation, migration and 


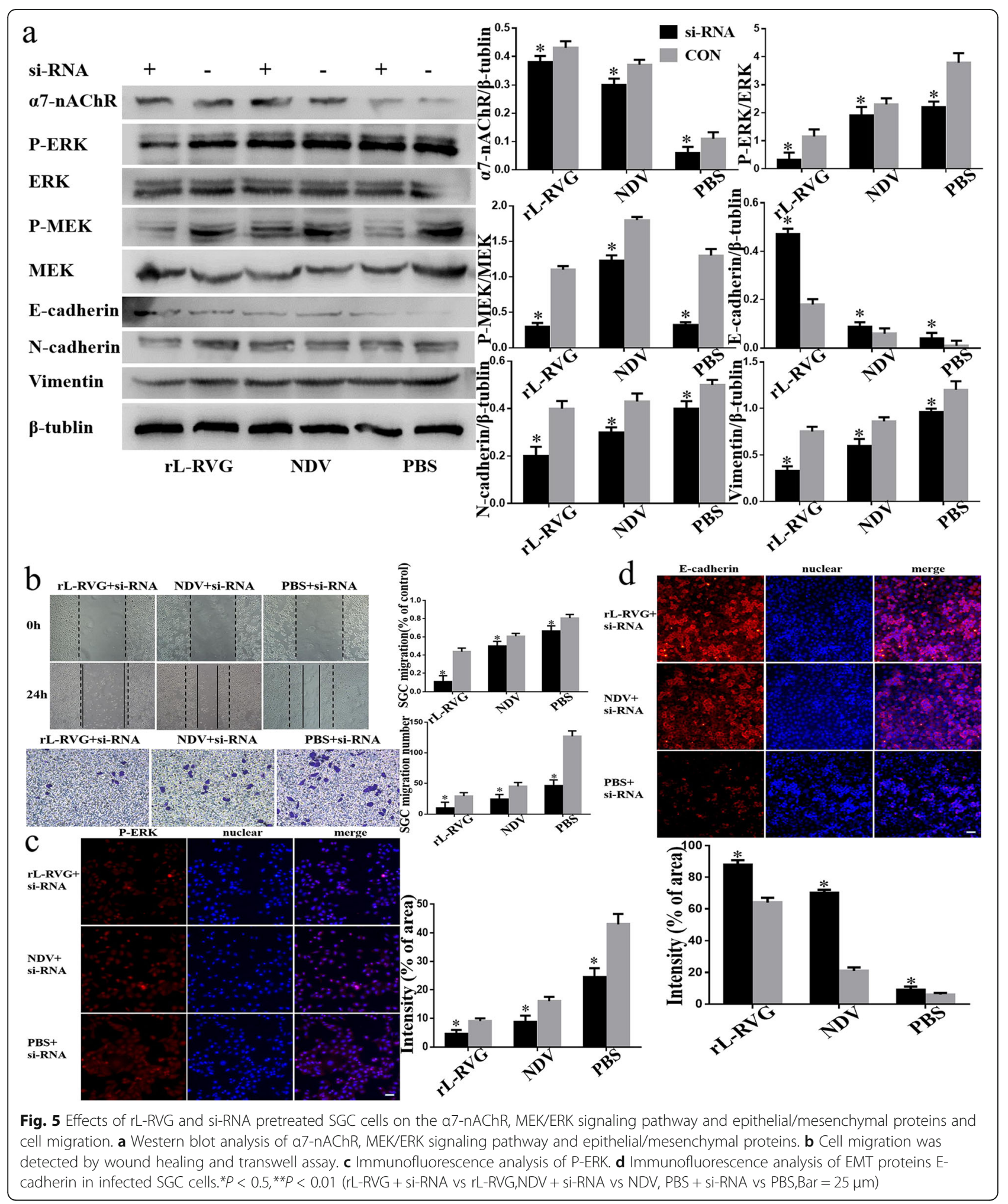

apoptosis of cells $[28,29]$. Therefore, inhibiting the ERK signaling pathway strengthens the anti-tumor activity of gimatecan in gastric cancer [30]. Activation of ERK signaling results in the promotion of cervical cancer cell growth and metastasis [31]. Interestingly, we found that rL-RVG reduces the phosphorylation levels of MEK/ERK and resulted in a decrease of phosphorylation levels of MLA, $\alpha 7$ $n A C h R$ and si-RNA pre-treated groups compared to the 


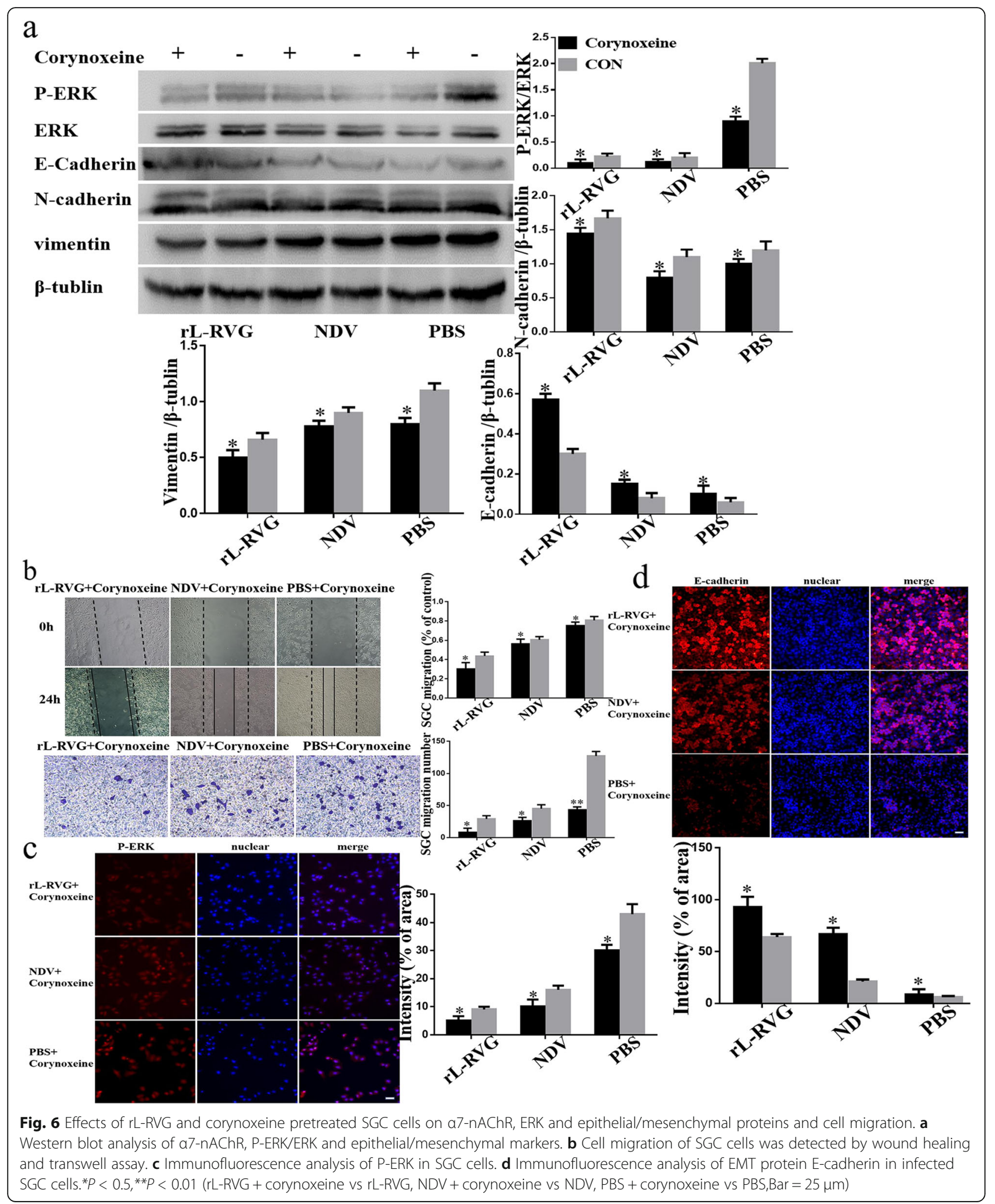

ACB pre-treated group. Additionally, we also achieved similar results in the change of EMT and migration of SGC cells. Hawsawi $\mathrm{O}$ and Henderson V suggested that HMGA2 may induce EMT via ERK signaling pathways [32]. In our study, after pretreatment with corynoxenine, we found that the expression of $\mathrm{N}$-cadherin and Vimentin was down- 


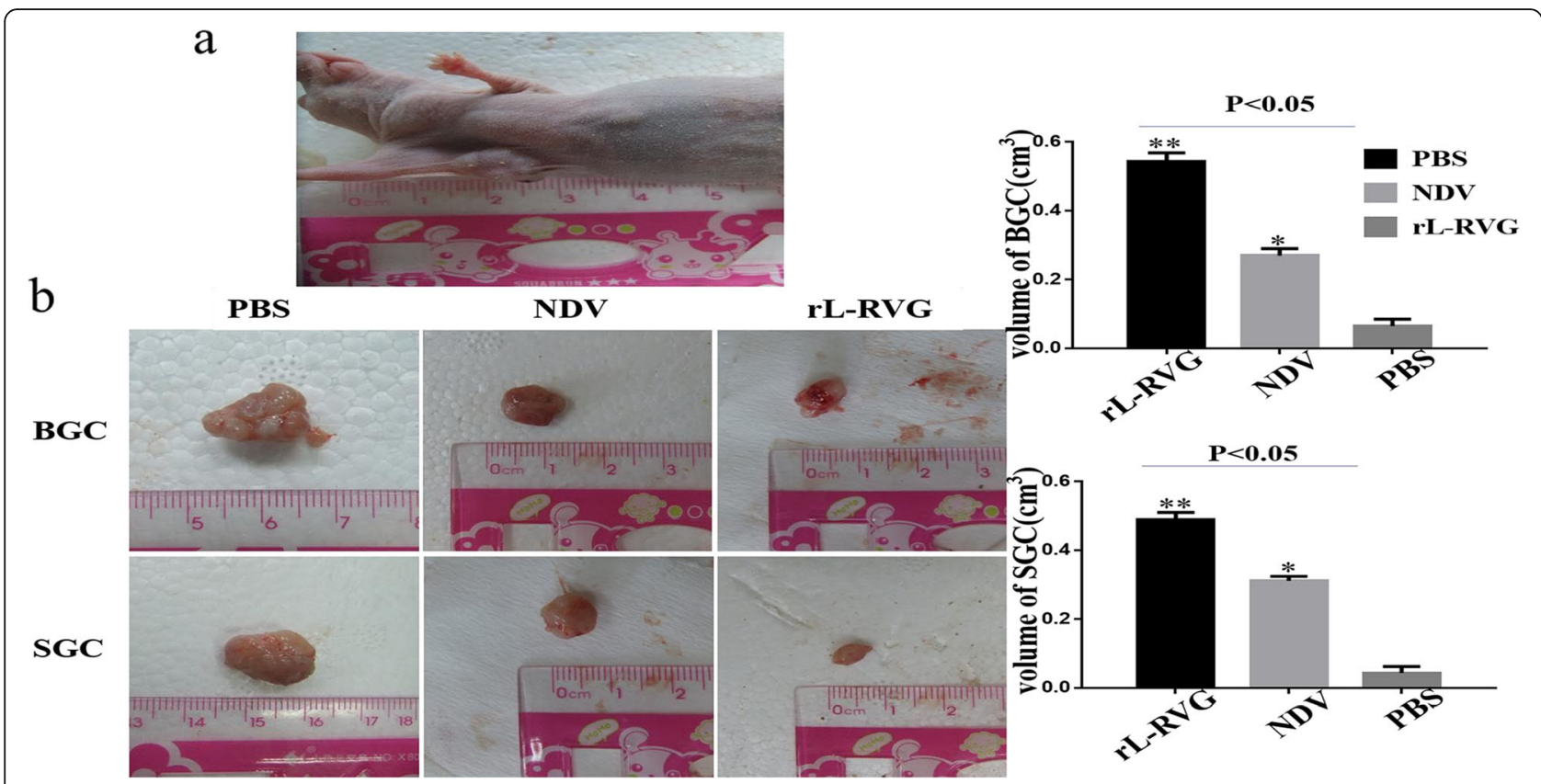

Fig. 7 rL-RVG suppressed the growth of subcutaneous tumor of BGC and SGC cells in vivo. a The subcutaneous tumor model was successfully established. $\mathbf{b}$ BGC and SGC cells were pretreated with rL-RVG, NDV or PBS for $24 \mathrm{~h}$ and then subcutaneously injected into axillary subcutaneous tissues of adult female athymic-nude mice. The subcutaneous tumor was examined after two weeks post treatment. ${ }^{*} P<0.5,{ }^{* *} P<0.01$. (rL-RVG vs NDV and PBS groups, respectively)

regulated and the expression of E-cadherin was upregulated compared to non-pretreated groups. We also found that rL-RVG has a much more inhibitory effect than NDV. Thus, rL-RVG does not only have the same effect as NDV, but also plays a role as competitive antagonist to inhibit $\alpha 7$ nAChR.

\section{Conclusion}

Our study provides a new option in the treatment of gastric cancer and provides novel insights into the mechanisms of rL-RVG in gastric cancer development. For the first time we demonstrated that rL-RVG acts as a competitive antagonist of $\alpha 7-\mathrm{nAChR}$ to suppress the migration of gastric cancer cells through inhibiting $\alpha 7$ nAChR-MEK/ERK-EMT.

\section{Supplementary information}

Supplementary information accompanies this paper at https://doi.org/10. 1186/s12885-019-6225-9.

Additional file 1: Result of STR matching analysis by your data.

\section{Abbreviations}

ACB: Acetylcholine block the nAChR DMSO:Dimethyl sulfoxide; CCK-8: Cell Counting Kit-8; ECL: Enhanced chemiluminescence; EGF: Epidermal growth factor; EMT: Epithelial-mesenchymal transition; ERK: Extracellular signalregulated kinase; MEK: Mitogen-activated protein kinase kinase; nAChRs: Nicotinic acetylcholine receptors; NDV: Newcastle disease virus; NNK: 4-(Methylnitrosamino)-1-(3-pyridyl)-1-butanone; PBS: phosphate buffered solution; P-ERK: Phosphorylated extracellular signal-regulated kinase;
P-MEK: Phosphorylated Mitogen-activated protein kinase kinase; PVDF: Polyvinylidinedifluoride; rL-RVG: Recombinant avirulent Newcastle disease virus (NDV) LaSota strain expressing the rabies virus glycoprotein: RPMI: Roswell park memorial institute; RVG: Rabies virus glycoprotein; SDS: Sodium dodecyl sulfate; TBST: Tris-Buffered Saline Tween-20; a7nAChR: alpha7 nicotinic acetylcholine receptor

\section{Acknowledgements}

The authors would like to thank to Zhijian Zhang, the Jiang Su University, China for their excellent experimental assistance. We thank the laboratory team for its collaboration.

\section{Authors' contributions}

$X B$ and $A Z$ participated in the conception and design of the study, data analysis,interpretation and manuscript writing. $C Y, Z C$ and $X Z$ collected and assembled the data. $A Z, R Z, Y Z$, and $J Z$ analyzed the data. $X B$ and $Y Y$ conceived the study. All authors read and approved the final manuscript.

\section{Funding}

This research topic came from the project of National Natural Science Foundation of China (No. 81672999), the study collection,analysis,and interpretation of data were subsidized by it. The authors declare no conflicts of interest.

\section{Availability of data and materials}

All data generated or analyzed during this study are included in this published article (and its Additional files). The datasets are available from the corresponding author upon reasonable request.

\section{Ethics approval and consent to participate}

All procedures in this study were approved and reviewed in advance by the Laboratory Animal Management Committee of Jiangsu University and also met the guidelines of the National Institutes of Health Guide for the Care and Use of Laboratory Animals. Ethical written consent of animal experiment was obtained. No ethical approval is required for using any of the cell lines employed for our study reported in this research article. 


\section{Consent for publication}

Not applicable.

\section{Competing interests}

The authors declare that they have no competing interests.

\section{Author details}

'Department of General Surgery, Affiliated People's Hospital of Jiangsu University, DianLi Road No.8, Zhenjiang 212002, JiangSu, China. ${ }^{2}$ Department of Liver and gallbladder surgery, First People's Hospital of Kunshan, QianJin West Road No.91, Kunshan 215300, JiangSu, China. ${ }^{3}$ Clinical Medicine College of Jiangsu University, XueFu Road No.301, Zhenjiang 212013, JiangSu, China. ${ }^{4}$ Department of General Surgery, Affiliated Hospital of Jiangsu University, JieFang Road No.438, Zhenjiang 212001, JiangSu, China. ${ }^{5}$ Department of Respiratory Medicine, Affiliated People's Hospital of Jiangsu University, DianLi Road No.8, Zhenjiang 212002, JiangSu, China.

Received: 4 January 2019 Accepted: 9 October 2019

Published online: 22 October 2019

\section{References}

1. Bray F, Ferlay J, Soerjomataram I, Siegel RL, Torre LA, Jemal A. Global cancer statistics 2018: GLOBOCAN estimates of incidence and mortality worldwide for 36 cancers in 185 countries. CA Cancer J Clin. 2018;68(6):394-424.

2. Chen W, Zheng R, Baade PD, Zhang S, Zeng H, Bray F, Jemal A, Yu XQ, He J. Cancer statistics in China, 2015. CA Cancer J Clin. 2016;66(2):115-32.

3. Jin X, Zhu Z, Shi Y. Metastasis mechanism and gene/protein expression in gastric cancer with distant organs metastasis. Bull Cancer. 2014;101(1):E1-12.

4. Millar NS, Gotti C. Diversity of vertebrate nicotinic acetylcholine receptors, Neuropharmacology. 2009;56(1):237-46.

5. Wessler I, Kirkpatrick CJ. Acetylcholine beyond neurons: the non-neuronal cholinergic system in humans. Br J Pharmacol. 2008;154(8):1558-71.

6. Schuller HM. Neurotransmitter receptor-mediated signaling pathways as modulators of carcinogenesis. Prog Exp Tumor Res. 2007:39:45-63.

7. Pavlov VA, Ochani M, Yang LH, Gallowitsch-Puerta M, Ochani K, Lin X, Levi J, Parrish WR, Rosas-Ballina M, Czura CJ, Larosa GJ, Miller EJ, Tracey KJ, Al-Abed Y. Selective a7-nicotinic acetylcholine receptor agonist GTS-21 improves survival in murine endotoxemia and severe sepsis. Crit Care Med. 2007;35(4): 1139-44.

8. Grando SA. Connections of nicotine to cancer. Nat Rev Cancer. 2014;14(6): 419-29.

9. Xu J, Liu D, Niu H, Zhu G, Xu Y,Ye D, Li J, Zhang Q. Resveratrol reverses Doxorubicin resistance by inhibiting epithelial-mesenchymal transition (EMT) through modulating PTEN/Akt signaling pathway in gastric cancer. J Exp Clin Cancer Res.2017;36(1): 19.

10. Yang Y, Zhang J, Yan Y, Cai H, Li M, Sun K, Wang J, Liu X, Wang J, Duan X. Low expression of Rap1GAP is associated with epithelial-mesenchymal transition (EMT) and poor prognosis in gastric cancer. Oncotarget.2017;8(5): 8057-8068. [11] Carino A, Graziosi L, D'Amore C, Cipriani S, Marchianò S, Marino E, Zampella A, Rende M1, Mosci P, Distrutti E, Donini A, Fiorucci S. The bile acid receptor GPBAR1 (TGR5) is expressed in human gastric cancers and promotes epithelial-mesenchymal transition in gastric cancer cell lines. Oncotarget.2016;7(38):61021-61035.

11. Lien YC, Wang W, Kuo LJ, Liu JJ, Wei PL, Ho YS, Ting WC, Wu CH, Chang YJ. Nicotine promotes cell migration through alpha7 nicotinic acetylcholine receptor in gastric cancer cells. Ann Surg Oncol. 2011;18(9):2671-9.

12. Zhang C, Ding XP, Zhao QN, Yang XJ, An SM, Wang H, Xu L, Zhu L, Chen $H Z$. Role of a7-nicotinic acetylcholine receptor in nicotine-induced invasion and epithelial-to-mesenchymal transition in human non-small cell lung cancer cells. Oncotarget. 2016;7(37):59199-208.

13. Bu X, Zhao Y, Zhang Z, Wang M, Li M, Yan Y. Recombinant Newcastle disease virus (rL-RVG) triggers autophagy and apoptosis in gastric carcinoma cells by inducing ER stress. Am J Cancer Res. 2016;6(5):924-36.

14. Yan $Y$, Su C, Hang M, Huang H, Zhao Y, Shao X, Bu X. Recombinant Newcastle disease virus rL-RVG enhances the apoptosis and inhibits the migration of A549 lung adenocarcinoma cells via regulating alpha 7 nicotinic acetylcholine receptors in vitro. Virol J. 2017;14(1):190.

15. Yan Y, Liang B, Zhang J, Liu Y, Bu X. Apoptotic induction of lung adenocarcinoma A549 cells infected by recombinant RVG Newcastle disease virus (rL-RVG) in vitro. Mol Med Rep. 2015;11:317-26.
16. Balakrishnan M, George R, Sharma A,Graham DY. Changing trends in stomach cancer throughout the world. Curr Gastroenterol Rep.2017;19(8):36. [18] Matsuoka T, Yashiro M. Biomarkers of gastric cancer: Current topics and future perspective. World J Gastroenterol.2018;24(26): 2818-2832.

17. LaRocca CJ, Warner SG. Oncolytic viruses and checkpoint inhibitors: combination therapy in clinical trials. Clin Transl Med. 2018;7(1):35.

18. Chulpanova D, Solovyeva V, Kitaeva K, Dunham SP, Khaiboullina SF, Rizvanov AA. Recombinant Viruses for Cancer Therapy. Biomedicines.2018; 6(4). pii: E94.

19. Krais AM, Hautefeuille AH, Cros MP, Krutovskikh V, Tournier JM, Birembaut $P$, Thépot A, Paliwal A, Herceg Z, Boffetta P, Brennan P, Hainaut PL. CHRNA5 as negative regulator of nicotine signaling in normal and cancer bronchial cells: effects on motility, migration and p63 expression. Carcinogenesis. 2011;32(9):1388-95.

20. Wang W, Chin-Sheng H, Kuo L J, Wei PL, Lien YC, Lin FY, Liu HH, Ho YS, Wu $\mathrm{CH}$, Chang YJ. NNK enhances cell migration through a7-nicotinic acetylcholine receptor accompanied by increased of fibronectin expression in gastric cancer. Ann Surg Oncol.2012;19 Suppl 3:S580-588.

21. Chernyavsky Al, Shchepotin IB, Grando SA. Mechanisms of growthpromoting and tumor-protecting effects of epithelial nicotinic acetylcholine receptors. Int Immunopharmacol. 2015;29(1):36-44.

22. Neuzillet C, Tijeras-Raballand A, de Mestier L, Cros J, Faivre S, Raymond E. MEK in cancer and cancer therapy. Pharmacol Ther. 2014;141(2):160-71.

23. Li T, Huang H, Shi G, Zhao L, Li T, Zhang Z, Liu R, Hu Y, Liu H, Yu J, Li G. TGF- $\beta 1$-SOX9 axis-inducible COL10A1 promotes invasion and metastasis in gastric cancer via epithelial-to-mesenchymal transition. Cell Death Dis. 2018; 9(9):849.

24. Peng Z, Wang CX, Fang EH, Wang GB, Tong Q. Role of epithelialmesenchymal transition in gastric cancer initiation and progression. World J Gastroenterol. 2014;20(18):5403-10.

25. Zhao K, He J, Wang Y, Jin SD, Fan Y, Fang N, Qian J, Xu TP, Guo RH. EZH2mediated epigenetic suppression of EphB3 inhibits gastric cancer proliferation and metastasis by affecting E-cadherin and vimentin expression. Gene. 2018;686:118-24.

26. Liang L, Zeng M, Pan H, Liu H, He Y. Nicotinamide N-methyltransferase promotes epithelial-mesenchymal transition in gastric cancer cells by activating transforming growth factor- $\beta 1$ expression. Oncol Lett. 2018;15(4): 4592-8.

27. Bu XF, Wang MB, Zhang ZJ, Zhao YH, Li M, Yan YL. Autophagy is involved in recombinant Newcastle disease virus (rL-RVG)-induced cell death of stomach adenocarcinoma cells in vitro. Int J Oncol. 2015;47(2):679-89.

28. Sun Y, Liu WZ, Liu T, Feng X, Yang N, Zhou HF. Signaling pathway of MAPK ERK in cell proliferation, differentiation, migration, senescence and apoptosis. J Recept Signal Transduct Res. 2015;35(6):600-4.

29. Huo Y N, Chen W, Zheng XX. ROS, MAPKJERK and PKC play distinct roles in EGF-stimulated human corneal cell proliferation and migration. Cell Mol Biol (Noisy-le-grand).2015; 61(7): 6-11.

30. Chen Z, Liu Z, Huang W, Li Z, Zou J, Wang J, Lin X, Li B, Chen D, Hu Y, Ji J, Gao J, Shen L. Gimatecan exerts potent antitumor activity against gastric cancer in vitro and in vivo via AKT and MAPK signaling pathways. J Transl Med. 2017;15(1):253.

31. Yang P, Su C, Luo X, Zeng H, Zhao L, Wei L, Zhang X, Varghese Z, Moorhead JF, Chen Y, Ruan XZ. Dietary oleic acid-induced CD36 promotes cervical cancer cell growth and metastasis via up-regulation Src/ERK pathway. Cancer Lett. 2018;438:76-85.

32. Hawsawi O, Henderson V, Burton $\sqcup$, Dougan J, Nagappan P, Odero-Marah V. High mobility group A2 (HMGA2) promotes EMT via MAPK pathway in prostate cancer. Biochem Biophys Res Commun. 2018;504(1):196-202.

\section{Publisher's Note}

Springer Nature remains neutral with regard to jurisdictional claims in published maps and institutional affiliations. 